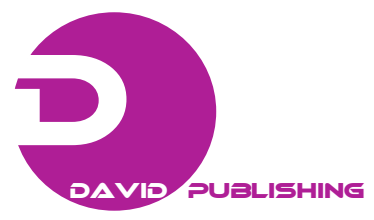

\title{
Fate of the Escherichia Coli Contaminated into Soil or Irrigation Water
}

\author{
Yasuhiro Inatsu* ${ }^{1}$, Rachel Ramos Elano ${ }^{2}$, Thongsavath Chanthasombath ${ }^{3}$, Borarin Buntong ${ }^{4}$ and Md Latiful Bari ${ }^{5}$ \\ 1. Food Hygiene Laboratory, National Food Research Institute, NARO, 2-1-12, Kannondai, Tsukuba, Ibaraki 305-8642, Japan \\ 2. National Food Authority Food Development Center, Philippines \\ 3. Clean Agriculture Development. Centre, Lao PDR \\ 4. Faculty of Agro-Industry, Royal University of Agriculture, Cambodia, \\ 5. Center for Advanced Research in Sciences, University of Dhaka, Bangladesh \\ *Corresponding author's e-mail: inatu@affrc.go.jp
}

\begin{abstract}
Pathogenic bacteria contaminated into raw vegetables have been recognized as a risk of foodborne illnesses. Recent researches have pointed that the contamination of these bacteria may be occurred from irrigation water, agricultural materials (such as manure) or entrance of animals into farms. The adaptation of good agricultural practices (GAP) is thought to be effective to reduce the risk of the contamination. However it is difficult to reveal the exact effectiveness of GAP on increasing the safeness of the outputs. The systematic collecting and analysis of field samples may give good evidence of it. However, it is rather difficult to conduct this work especially in developing countries due to the lack of research resources. Conducting suitable preliminary studies may helpful to reduce cost and man power required for experiments. From this view point, authors have performed pilot study about the fate of Escherichia (E.) coli artificially contaminated into several kinds of soils or water with these soils (as a model of irrigation water). 80 E. coli strains isolated from Japanese meat and vegetable samples (which showed different RAPD-PCR pattern) was contaminated into $100 \mathrm{~g}$ of each of 4 kind of soils or $100 \mathrm{~mL}$ of water that contained $5 \%(\mathrm{w} / \mathrm{v})$ of each of these soils. The $\mathrm{pH}$ and water contents of soils were 6.2-6.6 and $14 \%-31 \%$, respectively. These samples were leaved outside $\left(27-33^{\circ} \mathrm{C}\right)$ during July to September 2011 . The log reduction of inoculated E. coli in water (6.5 $\log \mathrm{CFU} / \mathrm{mL}$ ) after 4 weeks was 4.1 to $1.8 \log \mathrm{CFU} / \mathrm{mL}$. The log reduction of inoculated E. coli in soil (3-6 log $\mathrm{CFU} / \mathrm{g}$ ) was 0.5 to $2.9 \mathrm{log} \mathrm{CFU} / \mathrm{g}$ in the same period. No relationship with the log reduction and initial load of strains was found. From these results, keeping collected field samples in hot environment should be avoided to prevent the change of original contamination level of bacteria.
\end{abstract}

Key words: E. coli, contamination, soil, irrigation water, GAP. 